\title{
Erosão esternal por aneurisma da aorta. Qual o melhor acesso?
}

\author{
Roberto Gomes de CARVALHO*, Paulo R. GIUBLIN**, Luiz R. LOPES***, José Carlos MULASKI***, \\ Arleto Zacarias SILVA Jr. ${ }^{* *}$, Leonardo A. MULINARI ${ }^{* * *}$
}

RBCCV 44205-531

\begin{abstract}
Carvalho R G, Giublin P R, Lopes L R, Mulaski J C, Silva Jr A Z, Mulinari L A - Erosão esternal por aneurisma da aorta. Qual o melhor acesso? Rev Bras Cir Cardiovasc 2001; 16(1): 58-61.

RESUMO: É descrito o caso de um paciente que apresentava erosão esternal ocasionada por aneurisma da aorta ascendente e hemi-arco aórtico proximal. A via de acesso foi toracotomia bilateral uma vez que a erosão era na parte alta do esterno, empregando-se hipotermia profunda e parada circulatória total. Foi interposto enxerto de pericárdio bovino para correção da aorta ascendente e hemi-arco aórtico proximal e outro enxerto entre o tronco braquicefálico e a parede lateral do enxerto de pericárdio bovino. A artéria descendente anterior foi revascularizada por haver oclusão do óstio da artéria coronária esquerda. Após 3 anos da operação o paciente está assintomático.
\end{abstract}

DESCRITORES: Aneurisma da aorta torácica, cirurgia. Esterno, cirurgia. Esterno, patologia. Aneurisma de aorta torácica, complicações. Aorta, cirurgia.

\section{INTRODUÇÃO}

Erosão completa de esterno causada por aneurisma da aorta é uma situação incomum, sendo que a etiologia mais freqüente é a sífilis (1). O primeiro caso foi relatado em 1951 por COWLEY et al. (2). A questão é a via de acesso, pois a esternotomia ampla poderá levar ao sangramento com possível exsanguinação do paciente e também à mediastinite segundo BALAKRISHNAN \& KOPPULA ${ }^{(3)}$. Por estes motivos, são descritas diferentes técnicas de acesso (1-6). Os autores apresentam o caso de um paciente que apresentava erosão esternal causada por aneurisma da aorta ascendente e hemi-arco aórtico proximal e que a correção consistiu na interposição de um enxerto. O acesso foi por meio de toracotomia bi-lateral e hipotermia profunda com parada circulatória total e associada à revascularização do miocárdio.

\section{RELATO DE CASO}

Paciente do sexo masculino, branco, 36 anos, apresentando massa tumoral pulsátil e dolorosa medindo $4 \mathrm{~cm}$, localizada na região esternal ao nível do segundo espaço intercostal direito. Relatava dispnéia há 5 anos e doença sexualmente transmissível há 20 anos. Os exames para sífilis foram positivos (VDRL positivo com título de 1:128

Trabalho realizado no Serviço de Cirurgia Cardiovascular do Hospital Nossa Senhora das Graças. Curitiba, PR, Brasil.

Recebido para publicação em setembro de 2000.

* Do Hospital Nossa Senhora das Graças e Hospital Bichat (Paris)

** Da Policlínica de Pato Branco.

*** Do Hospital Nossa Senhora das Graças

Endereço para correspondência: Roberto G. de Carvalho. Rua Richard Strauss, 62. Curitiba, PR, Brasil. CEP 80820-110. e-mail: rcarvalho50@hotmail.com 
Carvalho R G, Giublin P R, Lopes L R, Mulaski J C, Silva Jr A Z, Mulinari L A. - Erosão esternal por aneurisma da aorta. Qual o melhor acesso? Rev Bras Cir Cardiovasc 2001; 16(1): 58-61.

e FTA-ABS positiva para IgG). Tomografia computadorizada de tórax mostrou erosão total do esterno provocada por uma das loculações do aneurisma (Figura 1A) e a angiotomografia tridimensional revelou o comprometimento da aorta ascendente e hemi-arco aórtico proximal (Figura 1B).

O acesso foi realizado por meio de toracotomia bilateral no quarto espaço intercostal e secção transversal do esterno. A instalação do circuito da circulação extracorpórea (CEC) foi realizada entre a artéria femoral e átrio direito e drenagem das cavidades esquerdas. A temperatura foi diminuída até $16^{\circ}$ $\mathrm{C}$ e realizada a parada circulatória total. $\mathrm{O}$ aneurisma foi aberto no sentido longitudinal, desde a porção acima dos óstios coronários até o hemi-arco aórtico proximal. Havia comprometimento da porção inicial do tronco braquiocefálico. O aneurisma apresentava
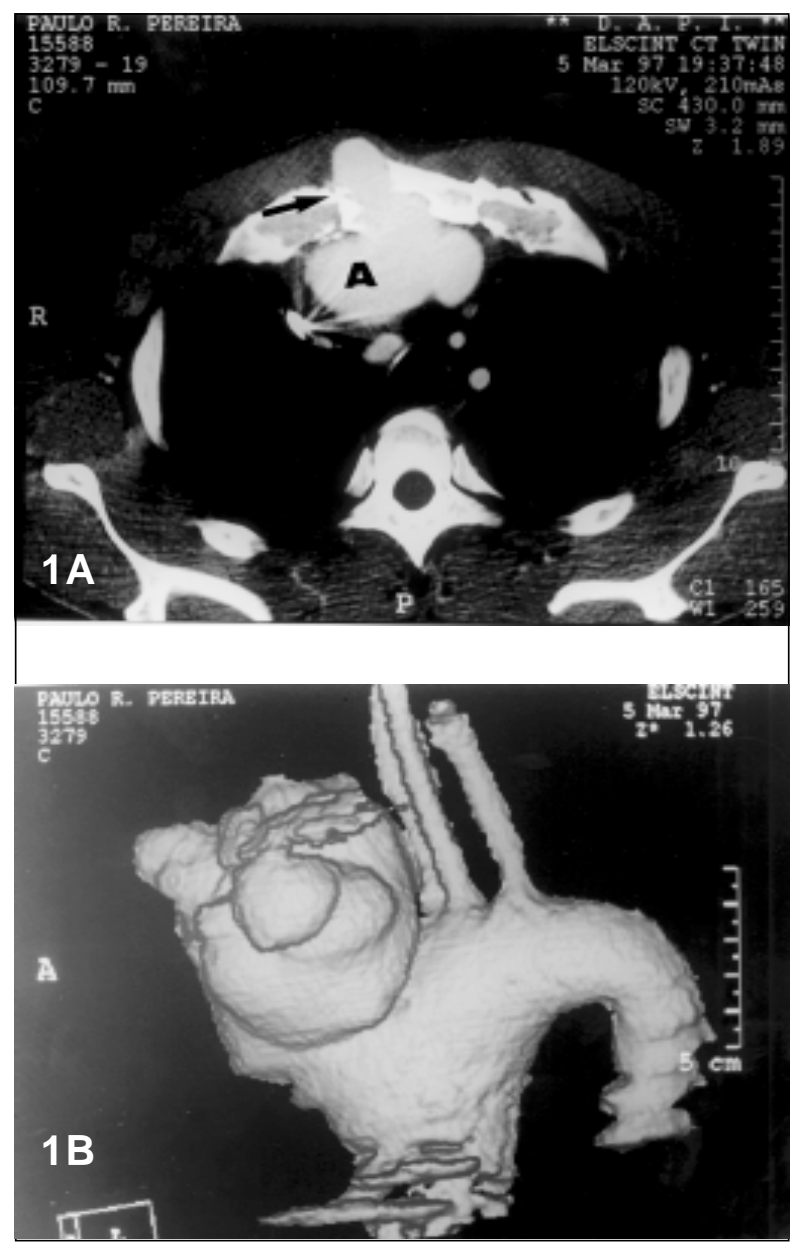

Fig. 1 A - Tomografia computadorizada de tórax demonstrando a erosão completa do esterno provocada pelo aneurisma. $A=$ aneurisma; flecha $=$ local da erosão

Fig. 1 B - Angiotomografia tridimensional onde se observa grande aneurysma da aorta com suas loculações dimensões $10 \times 15 \mathrm{~cm}$, sem trombos, contendo oito loculações e, uma delas, invadia o esterno levando à erosão. Foi observada oclusão total do óstio da artéria coronária esquerda (ACE), pela fibrose da íntima da aorta nesta região. O óstio da artéria coronária direita (ACD) não apresentava alterações. Proteção miocárdica foi realizada por meio de injeção de solução cardioplégica sangüínea no óstio da ACD. Enxerto corrugado de pericárdio bovino $31 \mathrm{~mm}$ (Braile Biomédica, São José do Rio Preto, SP, Brasil), foi suturado no arco aórtico, pouco antes da origem da artéria carótida comum esquerda. A seguir, um enxerto sintético de $14 \mathrm{~mm}$ (Hemashield - Meadox Medicals, Oakland, NJ, EUA), foi suturado entre a porção normal do tronco braquiocefálico e o enxerto de pericárdio bovino na sua face ântero-lateral direita. Este tempo cirúrgico foi realizado com parada circulatória total que durou 39 minutos. Em seguida, o enxerto de pericárdio bovino foi suturado de maneira término-terminal na aorta asccendente, logo acima do óstio da ACD. Foi realizada revascularização do miocárdio com ponte de safena para a artéria descendente anterior tenndo em vista que apresentava oclusão total do óstio da ACE.

Apresentou boa evolução pós-operatória. A angiotomografia tridimensional não demonstrou alterações e foi possível visibilizar a permeabilidade do enxerto aorto-coronário para a artéria descendente anterior (Figura 2). Após três anos da operação o paciente está assintomático e com vida normal.

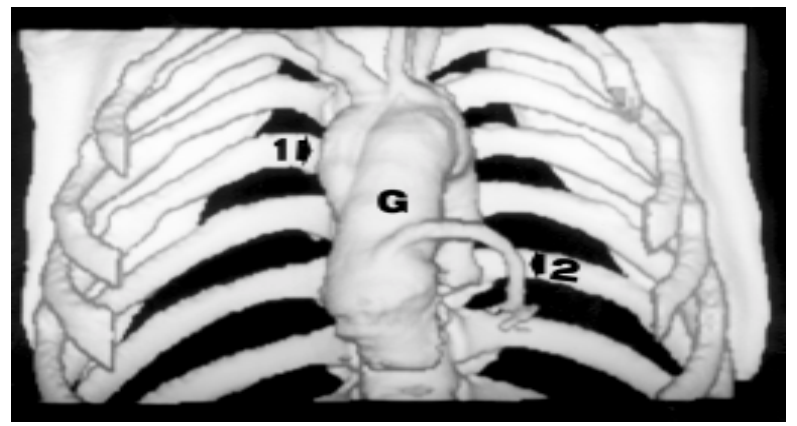

Fig. 2 - Angiotomografia tridimensional pós-operatória mostrando os dois enxertos e a ponte de safena pérvia. $G$ = enxerto corrugado de pericárdio bovino $(\mathrm{PB})$; flecha 1 = enxerto sintético entre tronco braquiocefálico e o enxerto de PB; flecha 2 = veia safena para artéria descendente anterior.

\section{COMENTÁRIOS}

A sífilis terciária pode comprometer a parede da aorta pela inflamação crônica e destruição de suas fibras elásticas e lisas. Como conseqüência, existe enfraquecimento de sua parede, levando à dilatação. A incidência do comprometimento aórtico é aproximadamente de $50 \%{ }^{(1)}$. 
Normalmente os aneurismas de origem sifilítica são saculares ou fusiformes. A sua dilatação pode comprimir estruturas vizinhas, e raramente pode existir a erosão total do esterno. O aneurisma pode estar localizado na aorta ascendente, hemi-arco aórtico proximal, como no presente caso e também descrito por FULTON et al. (1) e no hemi-arco aórtico distal, segundo KUBO et al. ${ }^{(4)}$.

Quando ocorre a erosão da parede torácica, significa que 0 aneurisma atinge grandes proporções e que, normalmente, uma das loculações provoca a erosão óssea. Este aspecto foi por nós observado na (Figura 1 A) e também por outros autores (1-6).

A dificuldade é escolher a via de acesso. Quando a CEC é instalada por via femoral, a drenagem venosa pode não ser suficiente como no caso descrito por FULTON et al. (1). Nesta situação foi necessário realizar esternotomia parcial e inferior. Deste modo, foi possível a canulação do átrio direito e a descompressão das cavidades esquerdas seguida de hiotermia profunda para realizar esternotomia ampla. Este acesso tem como desvantagem prolongar o tempo de hipotermia, bem como, a distensão das cavidades esquerdas, mesmo se houver insuficiência aórtica discreta.

Outra via de acesso foi descrita por TAKEUCHI et al. (5). Os autores realizaram a secção das clavículas e dos dois primeiros arcos costais, sendo que a incisão na pele foi em forma de "y". Assim, foi realizada esternotomia até o manúbrio e instalação da CEC com hipotermia profunda e parada circulatória total. Esta técnica de acesso pode levar à instabilidade da caixa torácica pela secção das clavículas e arcos costais. O conceito fundamental da minitoracotomia é justamente o oposto, ou seja, preservar a integridade da junção do esterno com a clavícula e arcos costais (7).

VANKER (6) descreveu um caso onde realizou toracotomia direita para instalação da CEC e hipotermia profunda. E após, durante esternotomia e secção do aneurisma com exsangüinação do paciente, foi realizada parada circulatória total e a sua correção. O motivo deste acesso era evitar a distensão do ventrículo esquerdo, pela presença de insuficiência aórtica leve. Este procedimento tem $\mathrm{o}$ inconveniente de prolongar o tempo de hipotermia profunda e parada circulatória, bem como, preparo do campo operatório.

A disponibilidade da drenagem com vácuo constitui alternativa útil para a instalação da circulação extracorpórea antes da toracotomia e conseguir drenagem adequada e evitar distenção.

No presente caso foi realizada toracotomia bilateral no quarto espaço intercostal. Este acesso facilita a instalação da CEC e a drenagem das cavidades esquerdas. Como não é realizada esternotomia, o local da destruição óssea não é manipulado. Deste modo não existe a possibilidade de mediastinite ou deiscência do esterno e, além disto, aorta ascendente e arco aórtico são bem acessíveis para abordagem cirúrgica, melhor que esternotomia. O defeito ósseo causado pela erosão não é tratado e. a longo prazo, não existe dificuldade para a cicatrização. Este acesso foi também realizado por BALAKRISHNAN \& KOPPULA (3).

Outro detalhe do presente caso foi o achado préoperatório da oclusão do óstio da ACE, também descrito por YAMADA et al. ${ }^{(8)}$, provocada pela sífilis. Cinecoronariografia não foi realizada porque o paciente não apresentava fatores de risco para doença arterial coronária e, eventualmente, poderia ocorrer complicações durante o procedimento, como rotura de uma das locuções. A dificuldade de desconexão da CEC por isquemia grave, decorrente da não perfusão da artéria coronária esquerda, levou à necessidade de revascularizar o miocárdio com ponte de veia safena para a artéria descendente anterior. Durante a operação observou-se que a parede da aorta era espessa, sendo remota a possibilidade de rotura durante a cinecoronariografia. Pela idade do paciente, poderia ser empregada a artéria radial para a revascularização do miocárdio. Mas optou-se pela veia safena, porque a retirada seria mais rápida, o paciente se encontrava por longo tempo em CEC e com instabilidade hemodinâmica grave.

\section{CONCLUSÕES}

A toracotomia bilateral é o acesso válido quando existe erosão esternal.

- A toracotomia bilateral melhora a exposição do campo operatório.

- A toracotomia bilateral facilita a estabilidade da CEC e drenagem das cavidades esquerdas.

- A toracotomia bilateral mantém a estabilidade do toráx pela integridade da junção esternoclavicular. 
Carvalho R G, Giublin P R, Lopes L R, Mulaski J C, Silva Jr A Z, Mulinari L A - Sternum erosion provoked by aortic aneurysm. Which is the better approach? Rev Bras Cir Cardiovasc 2001; 16(1): $58-61$.

ABSTRACT: The case of a patient presenting sternum erosion provoked by aneurysm of the ascending aorta and proximal hemi-arch of syphilitic etiology is described, the surgical approach was bilateral thoracotomy, using deep hypothermia and total circulatory arrest. A bovine pericardium patch was anchored for reapair of the ascending aorta and proximal aortic hemi-arch and another patch placed between the brachiocephalic trunk and the lateral wall of the bovine pericardium patch. The anterior interventricular artery was revascularized orving to occlusion of the ostium of the left coronary artery. Three years after surgery the patient is asymptomatic.

DESCRIPTION: Aortic aneurysm, thoracic, surgery. Sternum, surgery. Sternum, pathology. Aortic aneurysm, complications. Aorta, surgery.

\section{REFERÊNCIAS BIBLIOGRÁFICAS}

1 Fulton J O, Zilla P, de Groot K M, von Opell U O - Syphilitic aortic aneurysm eroding through the sternum. Eur $J$ Cardiothorac Surg 1996; 10: 922-4.

2 Cowley R A, Sloan H S, Sullenberger N H - Successful repair of an aortic aneurysm with sternal perforation. J Thorac Cardiovasc Surg 1951; 21: 159-63.

3 Balakrishnan K R \& Kopula A - Ascending aortic aneurysm eroding the chest wall: repair avoiding a median sternotomy [Letter]. J Thorac Cardiovasc Surg 1994; 107: $640-1$.

4 Kubo K, Hirose $\mathrm{H}$, Matsumoto K, Fuwa M, Mori $\mathrm{Y}$ Reoperation of a pseudoaneurysm eroding the sternum under hypothermic arrest of the brain through left thoracotomy. Nippon Kyobu Geka Gakkai Zasshi 1996; 44: 188-91.

5 Takeuchi E, Murash M, Tanaka M, Watanabe T, Usui A, Abe $T$ - New approach to the aneurysm originating in the ascending aorta, eroding the sternum and extending to the cervix. Nagoya J Med Sci 1988; 50: 27-34.

6 Vanker E A - A surgical approach to aortic arch aneurysms presenting through the sternum. $J$ Cardiovasc Surg (Torino) 1986; 27: 506-8.

7 Aris A - Reversed "C" ministernotomy for aortic valve replacement. Ann Thorac Surg 1999; 67: 1806-7.

8 Yamada T, Sakamoto T, Asano K et al. - Surgical treatment of syphilitic coronary ostial stenosis with aortic regurgitation. J Cardiovasc Surg (Torino) 1983; 24: 22-6. 